\title{
A pesquisa com EEG aplicada à área de aprendizagem motora
}

\author{
Caroline Luft \\ Alexandro Andrade
}

\author{
Universidade do Estado de Santa Catarina \\ Laboratório de Psicologia do Esporte e do Exercício \\ Florianópolis \\ Brasil
}

\section{RESUMO}

O eletroencefalograma (EEG) é o registro da atividade elétrica no córtex cerebral que corresponde ao fluxo de informações processado pelo córtex em suas atividades. O movimento envolve uma série de processos cognitivos específicos e a aprendizagem de um gesto passa por fases cognitivas que apresentam suas próprias características no encéfalo. O objetivo deste estudo foi realizar uma análise dos estudos com EEG dentro da pesquisa em aprendizagem motora, suas aplicações e possibilidades de estudos empíricos, visando colaborar com o crescimento desta área na Educação Física. Os principais estudos revisados indicam que a aprendizagem motora provoca uma mudança na ativação cortical na área pré-motora e motora do cérebro, ou seja, existe um aumento da atividade alfa (lenta e rítmica entre 8-12 Hz) nessas áreas, indicando que o gesto foi automatizado. Desta forma, a aprendizagem está relacionada a um menor esforço e maior eficiência neural, principalmente, na área pré-motora, pois esta é responsável pelo planejamento motor. Concluiu-se que o EEG pode ser uma excelente alternativa de pesquisa em aprendizagem motora, enriquecendo ainda mais os estudos nesta área, pela investigação mais direta dos processos cognitivos fundamentais envolvidos na habilidade motora e no seu aprendizado.

\section{ABSTRACT}

EEG and Motor Learning Research.

The Electroencephalogram (EEG) is the record of the cortex electrical activity that corresponds to the flow of information processed by the cortex during its activities. Physical movement involves specific cognitive processes and motor learning goes through by different phases and shows different characteristics in encephalon. The purpose of this paper was to review studies using EEG within the field of Motor Learning research, its applications and empirical possibilities. The main articles reviewed in this study, point out that motor learning causes a change in the cortical activation in the pre-motor and motor areas, $i . e$. , the alpha activity increases in these areas, indicating that the movement was learned. This way, motor learning is related to less effort and more neural efficiency mainly in the pre-motor cortex because this area is responsible for motor planning. Reviewing main results in empirical studies, we conclude that EEG may be an excellent tool in motor learning research, adding something more to this field studies by the direct investigation of the cognitive processes involved in motor ability and in its learning.

Key Words: electroencephalogram, motor learning, movement, brain cortex.

Palavras-chave: eletroencefalograma, aprendizagem motora, movimento, córtex cerebral. 


\section{INTRODUÇÃO}

A atividade elétrica do cérebro, resultante da comunicação eletroquímica entre seus neurônios, corresponde ao fluxo de informações e acontece em regiões distintas em função da atividade executada. Por exemplo, estados de alerta, sonolência e sono profundo apresentam diferentes características no encéfalo $(2,21)$.

O aprendizado é um conjunto de processos que provoca mudanças relativamente permanentes na capacidade de resposta $(2,9)$. A aprendizagem motora resulta em mudança, principalmente no comportamento motor. Por esta razão, é normalmente avaliada mediante a observação da performance em repetidas tarefas motoras no período de retenção. Porém, todo o aprendizado motor, resulta em mudanças na atividade neural em diferentes regiões do cérebro, principalmente nas áreas anteriores relativas à programação do movimento (7).

Estudos como os de Harris e Harris, Starkes e Wrisberg, citados por Haufler et. al. (14), sugeriram que o estilo de processamento de informação no cérebro (especialmente nos lobos frontais e temporais) de um atleta profissional apresenta maior eficiência, efetividade e menos esforço mental, quando comparado ao de novatos.

Existem muitos instrumentos de investigação da atividade neural no cérebro, como Topografia por Emissão de Positrons (PET), Representação funcional por Ressonância Magnética (RfRM) e Imagem Óptica (IO) (5). Na pesquisa em Aprendizagem Motora, o Eletroencefalograma (EEG) vem sendo o mais utilizado, pois além de ser um instrumento mais barato do que aqueles acima citados (23), pode registrar a atividade elétrica e as suas mudanças no cérebro, durante a execução de uma tarefa motora complexa ou durante o próprio exercício, com uma resolução temporal maior que a de outros instrumentos (10, 26). Embora o EEG apresente estas vantagens em relação aos outros instrumentos, existem dois fatores limitantes - de origem técnica e sociológica - do seu maior desenvolvimento e aplicação na mensuração do funcionamento cognitivo. A limitação técnica diz respeito à insuficiência de detalhes espaciais para identificar as estruturas e funções relacionadas à atividade elétrica, que podem ser visualizados com ressonância e outros métodos de neuroimagem. A limitação sociológica diz respeito ao fato de o EEG ter vindo a ser mais utilizado na área clínica, com fins diagnósticos, por médicos sem interesse em estudar o funcionamento cognitivo, enquanto na pesquisa é utilizado por professores de psicologia e seus estudantes, sem ambições clínicas, deixando a pesquisa um pouco distante da sua aplicação.

O EEG é o registro da atividade elétrica cerebral, em diferentes regiões do córtex, realizado através de eletrodos posicionados em regiões específicas no escalpo $(2,21)$.

A atividade rítmica no córtex é caracterizada por sua freqüência, que é a taxa na qual uma onda se repete dentro de 1 segundo, por isso é medida em hertz (ciclos por segundo) ou $\mathrm{Hz}$ (2). As freqüências mais comuns são: delta $(<4 \mathrm{~Hz})$, teta $(4-7 \mathrm{~Hz})$, alfa (8$13 \mathrm{~Hz})$ e beta $(>13 \mathrm{~Hz})(21)$. Podem ser encontrados valores diferentes, mas aproximados, em alguns estudos e também pode ser encontrada uma definição diferente que separa em ritmo alfa baixo $(9 \mathrm{~Hz})$ e alfa alto $(10-11 \mathrm{~Hz})(14)$. É importante saber essas definições básicas, porque diferentes freqüências, em regiões distintas, estão associadas com diferentes comportamentos.

As regiões a partir das quais os eletrodos são organizados no EEG são conhecidas por denominações abreviadas. A letra inicial corresponde à região - préfrontal $(\mathrm{Fp})$, frontal $(\mathrm{F})$, temporal $(\mathrm{T})$, central $(\mathrm{C})$, parietal $(\mathrm{P})$ e occipital $(\mathrm{O})$ - e o número ao hemisfério (ímpares: esquerdo, pares: direito). Quando a região vier seguida da letra $z$ é a área média da região, por exemplo: $\mathrm{Fz}, \mathrm{Cz}, \mathrm{Pz}$ e $\mathrm{Oz}$.

Cada estudo apresenta algumas particularidades metodológicas de investigação do EEG de uma tarefa específica. O EEG tem muitos canais e permite muitas montagens e derivações diferentes.

Normalmente, o objetivo de cada estudo define a montagem, a derivação e a frequiência a ser analisada. A maioria dos estudos investiga o EEG pela freqüência espectral (delta, teta, alfa, beta), embora existam formas mais específicas de investigação. Uma delas é pela mensuração dos macropotenciais cerebrais relacionados ao movimento (MCRMs) (7). Os MCRMs são investigados por EEG (Fpz, Fz, Cz, P3 e P4) coletados simultaneamente com eletromiografia (EMG) e separam cada fase cognitiva do gesto motor em períodos. O primeiro período é o pré- 
motor, que precede a performance motora. É caracterizado por um potencial negativo (deflexão para cima) que inicia 600-1200 ms antes da atividade muscular e é denominado Bereitschaftspotential (BP). Esse período corresponde à programação cognitiva do movimento.

O segundo período inicia com a contração muscular e continua até $80 \mathrm{~ms}$ após o pico do EMG e é chamado de período sensório-motor. Este é caracterizado por um pico negativo no potencial do córtex motor com uma latência de aproximadamente 100 ms (N100). O próximo período é identificado como período de finalização motora, caracterizado por um pico positivo, com latência de $200 \mathrm{~ms}$ (P200). E o último período é o pós-motor, caracterizado pela ocorrência de grande positividade com uma latência de 350-650 ms, conhecida como positividade de performance da habilidade (PPH) (7).

Os MCRMs mais estudados são os BP e PPH, pois neles são encontradas as principais diferenças entre novatos e profissionais, indicando que a aprendizagem motora altera principalmente a forma como o movimento é programado e também o feedback cerebral após o ato motor (7).

O período de latência de 300 ms nas regiões frontais, centrais e parietais, conhecido como P300, também é utilizado em pesquisas em aprendizagem motora. O P300 reflete o tempo de reação (TR) cerebral a um estímulo ambiental. Ele pode variar em amplitude e tempo (de 0 a $300 \mathrm{~ms}$ ) e, também, ser dependente do interesse pessoal pelo estímulo específico. Além de possibilitar uma medida direta do TR, o P300 pode, também, servir como um indicativo do grau de atenção do indivíduo, sendo útil em pesquisas em crianças com déficit de atenção por hiperatividade (DAH) (12).

O nível de atenção aos estímulos está diretamente relacionado com a aprendizagem motora. A aprendizagem também depende diretamente da memória, especialmente da de curto prazo (MCP), conhecida também como memória de trabalho (25). Em um estudo com neurofeedback (técnica que permite ao indivíduo controlar a própria atividade cerebral), foi sugerida a associação positiva entre atividade teta $\mathrm{e}$ memória de trabalho e, também, entre atividade do ritmo sensoriomotor (RSM), processamento da atenção e MCP.
Baseados em pesquisas que demonstraram que a MCP utiliza o córtex posterior de associação, envolvido no armazenamento de informação sensorial, e o córtex pré-frontal, que atualiza essa informação, Vernon e colaboradores (27) propuseram que essas duas regiões seriam conectadas pela atividade teta (4$7 \mathrm{~Hz}$ ). $\mathrm{O}$ treinamento da atividade teta se denomina Teta neurofeedback e tem o objetivo de melhorar a MCP. Neste mesmo estudo (27) foi sugerido que a atividade do RSM estaria diretamente relacionada com o processamento da atenção e que o treinamento desse ritmo facilitaria o aprendizado. O RSM é caracterizado por uma atividade rítmica nas regiões centrais (equivalentes às áreas sensório-motoras do cérebro) com uma freqüência entre $12-15 \mathrm{~Hz}$. O aparecimento da atividade RSM está diretamente relacionado a melhores níveis de processamento de atenção e de MCP.

Considerando que o EEG é pouco pesquisado na Educação Física no Brasil (ver periódicos nacionais), especialmente em Psicologia do Esporte e Aprendizagem Motora, e a possibilidade de investigação direta dos processos cognitivos envolvidos no gesto motor por meio do EEG, este estudo buscou fazer uma análise dos estudos sobre aprendizagem motora e EEG, como forma de embasar e estimular a realização de novos estudos empíricos sobre esse tópico.

\section{REVISÃO DA LITERATURA}

Os neurônios se comunicam de forma rápida e precisa, percorrendo longos trajetos. Mecanismos elétricos e químicos tornam possível a integração sináptica neural, permitindo que um neurônio forme mais de mil sinapses e receba mais de 10 mil conexões $(1,16)$. O sistema nervoso central é dividido em duas partes principais: encéfalo e medula espinhal. $O$ encéfalo está anatomicamente dividido em 3 partes: córtex, tronco encefálico e cerebelo $(1,16)$.

As áreas corticais individuais (sensorial, motora e cognitiva) são distinguidas pelas suas conexões de entrada e saída. Embora sejam diferentes, ambas as áreas são distribuídas da mesma maneira, organizadas em colunas verticais. A principal origem dos potenciais de EEG é a atividade elétrica das células piramidais, que têm como característica a projeção de seus axônios para outras áreas do cérebro e para 
a medula espinhal. Essas células são neurônios excitatórios, possuem axônios contralaterais que se projetam localmente e o seu principal neurotransmissor é o glutamato (18).

O uso do EEG nas pesquisas em Aprendizagem Motora pode ser enriquecedor, pois os processos cognitivos têm grande importância no aprendizado de uma habilidade e são constantemente mencionados em livros e estudos, porém, normalmente inferidos por meio de observação de comportamento e não investigados diretamente.

Aprendizado pode ser definido como um conjunto de processos associados com a prática ou experiência, que resulta em mudanças permanentes na capacidade de resposta (25).

A Aprendizagem Motora estuda a mudança no comportamento motor decorrente da aquisição de uma nova habilidade motora, que apresenta três características: a forma como o movimento é organizado, a importância relativa dos elementos motores e cognitivos e o nível de previsibilidade ambiental envolvendo performance e habilidade. A abordagem mais apropriada para classificar habilidade motora é a de considerar o grau com o qual os elementos cognitivos e motores contribuem para o alcance da meta com êxito. Por essa razão algumas vezes as habilidades motoras são chamadas de psicomotoras ou perceptomotoras (25).

Os aspectos cognitivos são essenciais na aquisição e na execução de novas habilidades motoras, por isso são fundamentais na Aprendizagem Motora. O cérebro é responsável por processos cognitivos que envolvem planejamento (córtex pré-frontal), organização da seqüência (pré-motor) e envio das ações específicas dos movimentos a serem executados (córtex motor). Outras áreas do cérebro também participam do movimento, enviando mensagens, dosando a força, agilidade, fornecendo feedbacks visuais, tácteis e auditivos, ajustando constantemente o movimento (16).

O planejamento e o aprendizado de uma habilidade motora provocam mudanças na atividade do cérebro, principalmente na sincronização dos impulsos neurais. A onda alfa, que é inversamente proporcional à ativação, está associada com uma maior sincronia e menor esforço neural, conseqüentemente, relacionada com melhor desempenho motor $(13,14)$.
A relação entre aprendizagem motora e cognição vem sendo alvo de muitos estudos. As mudanças decorrentes do aprendizado, e durante o mesmo, são normalmente mensuradas pela observação comportamental, avaliando a performance do indivíduo durante tarefas específicas, que são testadas subseqüentemente (período de retenção), constatando desta maneira se houve aprendizado (4).

Além da observação de comportamento, existem outras maneiras de investigar os processos cognitivos fundamentais no gesto motor. Uma delas é a utilização do EEG. O registro dos potenciais elétricos do cérebro associado com a execução de tarefas motoras específicas é um método útil para entender as funções corticais relacionadas à performance de movimentos voluntários (7).

Com o objetivo de investigar as mudanças neurofisiológicas associadas com o desenvolvimento de estratégias cognitivas e visuomotoras, Smith et al. (26) analisaram o EEG de dois grupos experimentais diferentes: o primeiro composto por 8 indivíduos durante duas tarefas diferentes de MCP (verbal e espacial) que requeriam um ajustamento constante, e o segundo com 6 indivíduos durante um jogo de video-game que continha um difícil componente visuo-motor. Os resultados demonstraram que a atividade alfa está diretamente relacionada com melhor performance motora, e que a sua localização no encéfalo aumenta em regiões específicas, dependendo da atividade realizada. A assimetria da atividade alfa também foi significativa neste estudo, indicando maior atividade alfa no hemisfério direito. A atividade alfa pode ser considerada um indício de aprendizado, uma vez que ela foi relacionada com atenção focalizada e com o desenvolvimento de estratégias cognitivas e visuomotoras eficientes.

Haufler et al. (14) realizaram um estudo no qual compararam a atividade do cérebro de atiradores novatos com o de profissionais durante a execução de uma prova de tiro (40 tiros), de uma tarefa de reconhecimento de palavras e de uma tarefa espacial. Constataram que a atividade eletroencefalográfica durante o tiro era significativamente menor (maior atividade alfa) nos profissionais do que nos novatos. Ao demonstrar pior performance, os novatos também apresentaram maior esforço mental e menor sincronia neural, especificamente nos lobos frontais, 
temporais e parietais. A atividade teta nos profissionais também foi significativamente maior durante a prova de tiro, o que indica que o nível de atenção deles era maior do que o dos novatos, pois esta onda está associada com o processamento da atenção. Nas outras tarefas não houve diferença significativa na atividade cerebral entre novatos e profissionais, sugerindo que o treinamento específico da habilidade resulta numa sincronia neural específica para a habilidade treinada.

Outra diferença funcional no cérebro, decorrente da aprendizagem, é a transferência da atividade neural. Durante a aquisição de uma nova informação, a atividade do cérebro é predominantemente maior no hemisfério direito e, depois do aprendizado, passa a ser maior no esquerdo; por essa razão, a atividade assimétrica pode também evidenciar a aprendizagem, ou não, de uma habilidade motora (11).

Etnier et al. (4) realizaram um estudo onde foi comparado o EEG de dois grupos, antes e depois da aquisição de uma nova habilidade motora. Um grupo realizou o pré-teste e treinou a habilidade (GE) durante o dia de aquisição, enquanto o outro fez o pré-teste, porém só executou a tarefa no outro dia durante o pós-teste (GC). O resultado encontrado foi que a performance do GE aumentou significativamente mais do que a do GC, bem como a atividade alfa no córtex. Não foram encontradas diferenças significativas entre a atividade nos hemisférios direito e esquerdo, sugerindo que o aprendizado está mais relacionado à atividade alfa do que com a ativação assimétrica do cérebro.

Hatfield et al. (13) realizaram um estudo de revisão, reunindo grande quantidade de estudos empíricos sobre aprendizagem motora e EEG. Foi verificado que a maioria dos estudos revisados indicaram que a aprendizagem motora resulta em aumento da atividade alfa (redução da ativação) nas regiões frontais, pré-frontais e temporais. Em relação à assimetria cortical, os estudos encontraram uma diminuição da atividade elétrica cortical no hemisfério direito, em relação ao esquerdo, nas regiões frontais, parietais e temporais, o que indica diferença significativa na assimetria antes e depois da aprendizagem. No estudo de Haufler et al. (14) foi verificado o aumento da atividade alfa (redução da ativação), especialmente no lobo temporal esquerdo.
O motivo pelo qual não se encontrou diferença significativa na assimetria cerebral no estudo de Etnier et al. (4) pode ter sido o de que a atividade eletroencefalográfica foi comparada apenas entre o hemisfério direito e esquerdo, não sendo consideradas diferenças entre as regiões dos hemisférios. Haufler et al. (14) compararam a atividade entre F3 - F4, T3 T4, P3 - P4, O1 - O2, e verificaram diferenças significativas na assimetria entre novatos e proficientes na tarefa de tiro, indicando que há diferenças, porém, elas não devem ser comparadas somente pela mensuração da atividade total dos hemisférios direito e esquerdo, e sim por diferentes regiões de ambos os lados (ex. assimetria frontal, temporal, parietal e occipital).

O estudo de Debaere et al. (3), que investigou a aprendizagem de uma tarefa bimanual, por meio de ressonância magnética, revelou diminuição da ativação no córtex, especialmente nas regiões corticais frontais do hemisfério direito (córtex dorsolateral pré-frontal, área pré-motora) e parietais de ambos os lados. Foram verificados aumentos na atividade na área motora primária, giro temporal superior, córtex motor cingulado, área pré-motora esquerda, entre outras áreas subcorticais.

Hatfield et al. (13) em seu estudo de revisão, sugerem que a aprendizagem provoca uma "economia de esforço", que aumenta a eficiência, ativando mais as áreas diretamente envolvidas com as demandas da tarefa e reduzindo a ativação das áreas que não são essenciais na sua realização, aumentando a eficiência. A teoria de Goldberg (11) apenas coloca que o hemisfério direito é responsável pelo processamento de estímulos novos, enquanto o esquerdo trabalha com informações assimiladas. Observando desta forma, cada região, em cada hemisfério, pode ter um padrão assimétrico diferente, em decorrência da aprendizagem.

$\mathrm{O}$ aumento da atividade rítmica no córtex, resultante da aprendizagem, pode ser verificado tanto interna quanto externamente. Pode-se verificar a presença de um determinado ritmo quando o movimento é automatizado, sendo esse ritmo diferente em cada indivíduo (24). Sakai et al. (2004) realizaram uma revisão sobre os estudos de ritmos internos da aprendizagem de habilidades motoras complexas com diferentes instrumentos de mensuração da atividade cerebral. 
Os estudos com PET indicaram que o movimento é aprendido em segmentos, sendo que a aprendizagem resulta na automatização de segmentos cada vez maiores. O córtex pré-frontal é responsável por selecionar os segmentos, colocando um após o outro. Portanto, quanto maior o tamanho do segmento, menor a demanda cognitiva. Essa sincronia neural resultante da aprendizagem seria a responsável pelo surgimento do ritmo neural. Quanto maior a aprendizagem, menor a demanda cognitiva, pois cada segmento é maior, conseqüentemente maior o ritmo neural (pode ser associado à atividade alfa). Fattapposta et al. (7) realizaram um estudo sobre como o tempo de prática influencia no aprendizado de uma nova habilidade motora. Investigaram os macropotenciais cerebrais relacionados ao movimento (MCRMs), entre eles: bereitschaftspotential (BP) que reflete os processos cerebrais referentes à programação do movimento e a positividade da performance da habilidade (PPH), que reflete os processos cerebrais de controle no resultado da atividade motora. A amostra foi constituída de 8 profissionais de tiro e 8 sujeitos sem prática de nenhum esporte. Foram executadas 4 sessões de 25 tentativas, sendo que a tarefa motora foi o tiro. Os resultados demonstraram diferenças significativas no BP relacionadas à performance, sendo que os profissionais apresentaram menor amplitude (o que está associado a menor esforço de programação de movimento), enquanto que os novatos demonstraram maior amplitude, que aumentava no decorrer das sessões, juntamente com uma melhora da performance. Esses resultados indicam que a BP entre novatos e profissionais difere, dependentemente do nível de aprendizado, sugerindo que durante a aquisição da habilidade a amplitude de BP aumenta relativamente ao nível de programação e atenção nos detalhes, e que, depois da automatização, a BP diminui, indicando menor esforço mental e o direcionamento da atenção apenas aos detalhes relevantes.

Existe a necessidade de mais estudos que comparem a atividade entre regiões e hemisférios cerebrais, que dividam e comparem as regiões utilizando os mesmos critérios (regiões, freqüências, análise estatística) com o intuito de possibilitar uma melhor compreensão de como o aprendizado influencia a atividade neural, bem como de permitir comparações confiáveis.
Além dos processos neurais referentes ao planejamento e automatização do movimento, a memória também é uma variável cognitiva fundamental na aprendizagem motora. A memória pode ser definida como a "capacidade do indivíduo de reter e utilizar a informação de várias maneiras por vários períodos de tempo" (25, p. 96). Ela pode ser conceituada também como "a armazenagem de material resultante das atividades dos vários estágios de processamento de informação" (p.96). Existem três sistemas distintos de memória envolvidos no processamento da informação, que resultam na produção de movimento: armazenamento sensorial de curto prazo (ASCP), memória de curto prazo (MCP) e memória de longo prazo (MLP). A MCP também é conhecida como memória de trabalho (25).

Vernon et al. (27) realizaram um estudo com a utilização de neurofeedback (técnica onde o indivíduo aprende como influenciar a atividade elétrica do seu cérebro, mediante um feedback constante que lhe é fornecido) relacionado a MCP. A pesquisa estava baseada na evidência, apontada por outros estudos $(9,15)$, de que a atividade teta $(4-7 \mathrm{~Hz})$ está associada positivamente com a MCP. A hipótese para esta relação é que o córtex de associação posterior (envolvido na retenção de informação sensorial) e o córtex pré-frontal (responsável por atualizar essa informação) seriam conectados pela atividade teta. Neste mesmo estudo (27) foi investigada a relação da supressão da atividade do ritmo sensoriomotor (RSM) $(12-15 \mathrm{~Hz})$ com o processamento da atenção. Os resultados encontrados foram que o grupo que treinou o RSM neurofeedback melhorou a atenção e também a MCP, enquanto que o grupo que treinou apenas a atividade teta não apresentou diferenças significativas. Sugeriu-se então que a memória de trabalho está mais associada com a atenção (relacionada ao RSM) do que com a atividade teta.

Outro estudo (15) investigou o neurofeedback do RSM em 530 crianças e adultos com problemas de atenção. Após 20 sessões de tratamento (RSM neurofeedback) o nível de atenção melhorou significativamente, bem como o tempo de reação e o controle da impulsividade. Este estudo propôs que o RSM está ligado diretamente aos níveis de atenção e sugeriu que o seu treinamento pode também, por este motivo, auxiliar na aprendizagem, principalmente de crianças com déficit de atenção por hiperatividade (DAH). 
Em outra investigação (17) foi pesquisado o comportamento de diferentes freqüências no cérebro de 21 indivíduos dentro de um simulador de direção em quatro situações: o simulador parado com o motor desligado no momento de largada (VCO), andando com o motor ligado a $45 \mathrm{mph}$ (sem interferência do sujeito experimental) (VC45), andando a $90 \mathrm{mph}$ (VC90) e dirigindo, ou seja, controlando o simulador (controle motor - CM). Os resultados demonstraram que, durante as tarefas visuais (VCO, VC45 e VC90), houve supressão bilateral da atividade $11-15 \mathrm{~Hz}$ localizada no córtex temporo-parietal, enquanto que os movimentos corporais produziram uma supressão similar no córtex somatosensorial médio. Esse resultado indica que a atividade motora está associada com uma supressão da atividade RSM, o que sugere a interferência do movimento nos componentes perceptuais e integrativos do processamento de informação.

McEvoy e Smith (20) testaram a confiabilidade de 4 freqüências de EEG durante a execução de uma tarefa motora de tempo de reação (TR) e de uma tarefa de MCP em diferentes níveis de dificuldade. As quatro ondas testadas foram: atividade teta frontal média $(\mathrm{Fm} \theta)$, teta posterior, alfa baixa, alfa alta, com freqüências e localização de respectivamente: $4-7 \mathrm{~Hz}$ em Fz, 4-7 Hz em Pz, média 9,5 Hz em $\mathrm{Pz}$ e Cz e média $11 \mathrm{~Hz}$ em $\mathrm{Pz}$ e Cz. Os resultados indicaram que quanto maior o nível de dificuldade, maior a Fm $\theta$ (atividade teta na região frontal média) e menor a atividade alfa. No entanto, a atividade teta posterior não apresentou diferença relativa à realização das tarefas. Através da análise estatística foi constatado alto nível de confiabilidade entre os resultados de EEG e as tarefas executadas, indicando que estas quatro freqüências de EEG são confiáveis para estudos de tarefas psicomotoras e de MCP. Outra variável de EEG muito utilizada em pesquisas em aprendizagem motora é o P300. O P300 refere-se ao período de tempo entre 0 e $300 \mathrm{~ms}$ onde há uma latência entre o estímulo e a reação no cérebro na região sensório-motora. O P300 serve para indicar o tempo de reação no cérebro. O P300 é muito usado para medir a atenção e a sua amplitude também varia de acordo com o estímulo e com a necessidade, ou não, de resposta (12).
O P300 também pode ser utilizado como um indicativo de atenção a um estímulo auto-relevante (12). Este estímulo auto-relevante, no estudo citado anteriormente (12), foram palavras que tinham relação com a vida do indivíduo, como nome dos pais, cidade natal, telefone, etc. Foi comparado o P300 para palavras neutras e relevantes. Os resultados indicaram que o P300 era maior em amplitude e menor em tempo de reação quando a palavra apresentada era relevante na vida do indivíduo. Este estudo indicou que os níveis de atenção aos estímulos externos variam de acordo com o valor atribuído ou com o grau de familiaridade a eles, sugerindo que a relação subjetiva do indivíduo-estímulo é importante na atenção e na reação ao mesmo.

\section{DISCUSSÃO}

Os estudos $(26,13,14)$ demonstraram que quanto maior a atividade alfa, maior a sincronia neural, menor o esforço mental e, conseqüentemente, melhor o desempenho. A atividade alfa é importante nas pesquisas em aprendizagem motora porque pode indicar se houve ou não aprendizado. Também foi encontrado que a atividade alfa aparece especificamente para a habilidade aprendida, pois outro estudo (14) demonstrou que profissionais e novatos não apresentaram diferença na atividade cortical durante a realização de tarefas com as quais não estavam familiarizados.

A assimetria da atividade alfa também sugeriu, nestes estudos, que hemisférios e regiões diferentes estão relacionadas ao aprendizado motor. A teoria de Goldberg (11) diz que o aprendizado acontece assimetricamente, sendo que, durante este processo, o hemisfério direito é mais ativo e, depois de concretizado, o esquerdo é que apresenta maior atividade no resgate da tarefa aprendida. Ou seja, depois do aprendizado, o hemisfério esquerdo apresenta maior ativação. Essa teoria não foi confirmada pelo estudo de Etnier et al. (4), pois neste não foi encontrada diferença significativa na assimetria cerebral depois da aprendizagem. Porém, deve-se considerar que este estudo (4) analisou a assimetria total do cérebro e não em diferentes regiões do mesmo. Outros estudos $(13,14$, 26) encontraram diferenças na assimetria em diferentes regiões relacionadas ao aprendizado. No primeiro (14), o lobo parietal direito mostrou maior atividade 
alfa em relação ao esquerdo (nos profissionais) e, considerando que quanto maior a atividade alfa, menor a ativação cerebral, o lobo parietal esquerdo estava mais ativo nos profissionais, confirmando a teoria de Goldberg (11). O outro estudo (26) também encontrou maior ativação no lobo esquerdo, em comparação com o direito (indicado pela atividade alfa), em regiões específicas requeridas pelas tarefas propostas no estudo. Ambos os estudos sugeriram que o EEG é válido para mensurar o aprendizado motor e estudar diferentes estratégias cognitivas envolvidas no processo. A teoria de Goldberg (11) pode ser complementada pela idéia de Hatfield et al. (2004) que relatam que o aprendizado é um processo específico para cada habilidade, sendo que as estruturas que não são diretamente responsáveis pela execução da atividade têm a sua atividade reduzida com a prática, provocando uma economia de energia, direcionando-a para as áreas mais importantes na execução da tarefa. Desta forma, a assimetria, da direita para a esquerda, aconteceria principalmente nas áreas diretamente envolvidas com a tarefa.

A principal diferença relativa ao aprendizado de uma habilidade foi com relação ao planejamento motor. A execução do movimento passa por 3 fases: planejamento cognitivo (córtex pré-motor), organização e envio das seqüências (córtex motor) (16). O estudo de Fattapposta et al. (7) demonstrou com os MCRMs que a amplitude do BP (macropotencial relacionado à programação do movimento) é menor em profissionais do que em novatos durante uma tarefa de tiro, indicando também menor esforço neural e maior concentração apenas em sinais relevantes do ambiente.

O resultado deste estudo (7), embora tenha usado um método diferente, pode ser relacionado com o anterior (14). Ambos os estudos compararam a atividade neural durante a execução de uma prova de tiro de novatos e profissionais. Porém, Haufler et al. (14) analisaram a atividade pela freqüência alfa e

Fattapposta et al. (7) pelos MCRMs. Mas, ambos encontraram resultados similares, que associam menor esforço neural nos profissionais, seja ele indicado pela maior atividade alfa ou por menor amplitude do BP. Estes estudos demonstram que a principal mudança promovida pelo aprendizado está na programação do movimento.
Outra variável importante na aprendizagem motora é a memória, especialmente a de curto prazo (MCP). Alguns estudos $(27,15,17)$ investigaram variáveis relacionadas à MCP. O primeiro (27) demonstrou que a MCP está mais associada à atividade do RSM do que a atividade teta na região frontal. Na outra investigação (15) foi demonstrado que o treinamento da atividade do RSM melhora significativamente os níveis de atenção, indicando que o RSM tem relação direta com a atenção. E o terceiro estudo (17) demonstrou que durante uma tarefa motora há uma supressão da RSM, indicando que o movimento diminui os níveis de atenção.

O tempo de reação também é uma variável importante para a aprendizagem motora e está relacionado também com os níveis de atenção. Foi demonstrado (12) que o tempo de reação pode ser medido diretamente através do P300. O tempo que o cérebro demora a reagir e a magnitude (amplitude) da reação não dependem apenas da natureza do estímulo, mas também do significado individual atribuído ao estímulo. Esse estudo (12) demonstrou que a história pessoal influencia nas respostas ao mesmo estímulo, fazendo com que o cérebro reaja mais e mais rápido a coisas que se associam com a sua vida. O maior problema na utilização do EEG é a dificuldade em associar os eventos elétricos corticais às estruturas, e a incapacidade de verificar a atividade das estruturas subcorticais na aprendizagem do movimento. Um estudo realizado com PET (19) verificou a importância do cerebelo no aprendizado motor implícito, atividade esta que não poderia ser verificada com EEG.

Nos estudos de revisão $(6,13)$ os resultados apontaram que a atividade cortical encontrada em investigações com EEG concordam com os achados por $\operatorname{PET}(6,19)$, ressonância $(3)$ e por mensurações eletrofisiológicas de atividade autônoma (22). Todos estes estudos apontam uma redução na atividade cortical decorrente da aprendizagem.

\section{CONCLUSÃO}

Pode-se concluir, com base nos estudos revisados, que a aprendizagem motora altera principalmente a atividade de programação motora e o feedback neural após o movimento. Além da alteração dos MCRMs, foi demonstrado que a investigação das freqüências 
de EEG é confiável para o estudo da aprendizagem motora $(14,17,20)$.

A investigação do P300 (12) levou à conclusão de que o significado do estímulo é essencial na resposta cerebral, pois demonstrou que palavras com significado pessoal apresentavam um P300 com maior amplitude e menor tempo de reação, ressaltando dessa forma a importância da subjetividade do sujeito na resposta. Uma das áreas de interesse dos pesquisadores deste estudo é a amplitude do P300 relacionada a estímulos mais significantes comparados aqueles não-significantes para o indivíduo. O significado do ambiente e dos estímulos que o compõem são fundamentais no estudo de uma psicologia que vise investigar o sujeito no processo de vivência do esporte, pois o movimento sempre tem uma finalidade, surgindo normalmente após uma avaliação subjetiva de significado (8).

Os estudos aqui revisados demonstraram que o EEG é uma boa alternativa para a pesquisa em aprendizagem motora e que permite a compreensão dos processos cognitivos envolvidos na aquisição de uma nova habilidade, bem como na execução proficiente de tarefas motoras. O EEG permite também atuar e compreender melhor o aprendizado em diferentes situações e indivíduos e apresenta alto nível de confiabilidade (27).

CORRESPONDÊNCIA

Alexandro Andrade UDESC/CEFID

Laboratório de Psicologia do Esporte e do Exercício Rua Pascoal Simone n’358

Florianópolis SC

BRASIL

d2aa@udesc.br 


\section{REFERÊNCIAS}

1. Bear MC, Barry W, Paradiso MA (2001). Neuroscience: Exploring The Brain. 2 Ed. Maryland: Lippincott Williams \& Wilkins.

2. Cantor DS (1999). An Overview of Quantitative EEG and its Applications to Neurofeedback. In: Evans JR, Abarbanel A. Introduction to Quantitative EEG and Neurofeedback. Copyright: Academic Press.

3. Debaere, F, Wenderoth, N, Sunaert, S, Hecke, PV, Swinnen, SP (2004). Changes in brain activation of a new bimanual coordination task. Neuropsychologia 42: 855-867.

4. Etnier JL, Whitwer SS, Landers DM, Petruzzello SJ, Salazar W (1996). Changes in Eletroencephalographic Activity Associated with Learning a Novel Motor Task. Research Quarterly for Exercise and Sport 67(3): 272-279.

5. Fabiani M, Gratton G, Corbalis PM (1996). Noninvasive near infrared optical imaging of human brain function with subsecond temporal resolution. Journal of Biomedical Optics 1: 387-398.

6. Fadiga, L, Craighero, L (2004). Electrophysiology of action representation. Journal of Clinical Neurophysiology 21 (3) 157-169.

7. Fattaposta F, Amabile G, Cordishi, MV, Venanzio D, Di Foti A, Pierelli F, Alessio CD, Pigozzi F, Parisi A, Morrocutti C (1996). Long-term pratice effects on a new skilled motor learning: an ekectrophysiological study. Electroencephalography and clinical Neurophysiology 99: 495507.

8. Feijó, Olavo G (1998). Psicologia para o Esporte. 2 ed. Rio de Janeiro: Shape Ed.

9. Gevins A, Smith ME, Mcevoy L, Yu D (1997). High resolution EEG mapping of cortical activation related to working memory: effects of task difficulty, type of processing and pratice. Cerebral Cortex 7: 374-385.

10. Gevins, A (1998). The future of electroencephalography in assessing neurocognitive functioning. Electroencephalography Clinical Neurophysiology 106 (2): 165-172.

11. Goldberg, E (2002). O cérebro executivo: Lobos Frontais e a Mente Civilizada. Rio de Janeiro: Ed. Imago.

12. Gray HM, Ambady N, Lowenthal WT, Deldin P (2003). P300 as an index of attention to self-relevant stimuli. Journal of Experimental Social Psychology. Academic Press (In press).

13. Hatfield BD, Haufler AJ, Hung TM, Spalding TW (2004). Electroencephalographic Studies of Skilled Psychomotor Performance. Journal of Clinical Neurophysiology 21 (3): 144156.

14. Haufler AJ, Spalding DL, Santa Maria DL, Hatfield BD (2000). Neuro-Cognitive activity during a self-paced visuospatial task: comparative EEG profiles in marksmen and novice shooters. Biological Psychology 53: 131-160.

15. Kaiser DA, Othmer S (1997). Efficacy of SMR-Beta Neurofeedback for Attentional Processes. Inc. Encino CA. EEG Spectrum. November.

16. Kolb B, Whishaw IQ (2002). Neurociência do Comportamento. Barueri: Editora Manole Ltda.

17. Mann CA, Sterman M, Barry K, David A (1996). Supression of EEG rhythmic frequencies during somatomotor and visuo-motor behavior. International Journal of Psychophysiology 23: 1-7.

18. Martin, JH (1991). The Collective Electrical Behavior of Cortical Neurons: The Eletroencephalogram and the
Mechanisms of Epilepsy. In: Kandel ER, Schartz JH, Jessell TM Principles of Neural Science. 3 ed. Connecticut: Appleton \& Lange.

19. Matsumura M, Sadato N, Kochiyama T, Nakamura S, Naito E, Matsunami K, Kawashima R, Fukuta H, Yonekura Y (2004). Role of cerebellum in implicit motor skill learning: a PET study. Brain Research Bulletin 63: 471-483.

20. Mcevoy LK, Gevins A (2000). Test-Retest Reliability of Cognitive EEG. Clinical Neurophysiology 111: 457-463.

21. Montenegro MA, Cendes F, Guerreiro, M, Guerreiro, CA (2001). EEG na prática clínica. São Paulo: Lemos Editorial.

22. Oishi K, Maeshima T (2004). Autonomic nervous system activities during motor imagery in elite athletes. J Clin Neurophysiol 21 (3): 170-179.

23. Ross P. (2003). Leitores da Mente. Scientific American. Brasil 17: 66-68.

24. Sakai K, Hikosaka O, Nakamura K (2004). Emergence of rhythm during motor learning. Trends in Cognitive Sciences 8 (12): 547-553.

25. Schmidt RA, Wrisberg CA (2001). Aprendizagem e Performance Motora: Uma abordagem da aprendizagem baseada no problema. 2 ed. Porto Alegre: Artmed Editora Ltda.

26. Smith ME, Mcevoy LK. Gevins A (1999). Neurophysiological indices of strategy development and skill acquisition. Cognitive Brain Research 7: 389-404.

27. Vernon D, Egner T, Cooper N, Compton T, Neilands C, Sheri A, Gruzelier J (2003). The effect of training distinct neurofeedback protocols on aspects of cognitive performance. International Journal of Psychophysiology 47: 75-85. 